DEPÓSITO LEGAL ZU2020000153

Esta publicación científica en formato digital

es continuidad de la revista impresa

ISSN 0041-8811

E-ISSN 2665-0428

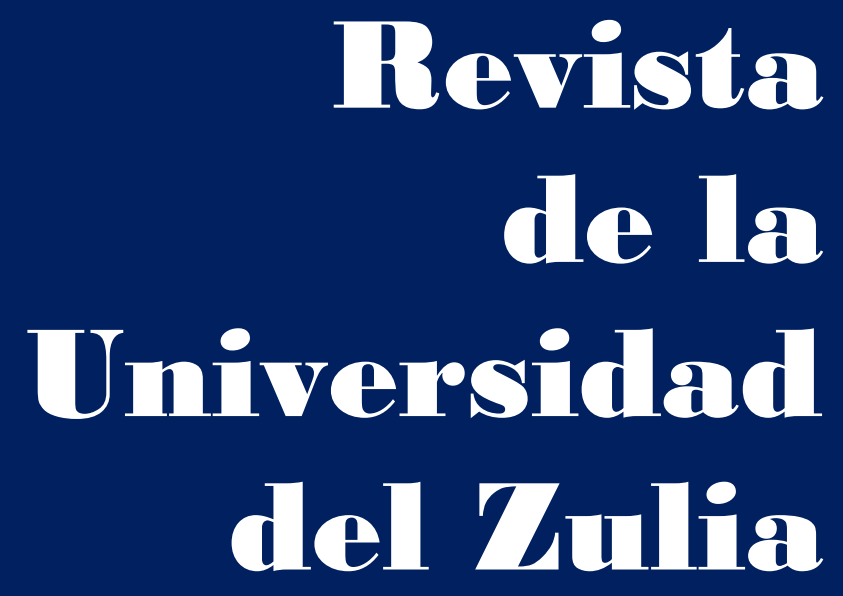

Fundada en 1947

por el Dr. Jesús Emrique Lossada

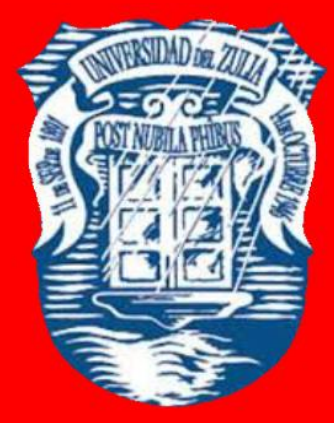

Ciencias

Sociales

y Arte

Año $12 \quad N^{\circ} 34$

Septiembre - Diciembre 2021

Tercera Época

Maracaibo-Veneruela 


\title{
Presupuesto participativo por resultados y la toma de decisiones en municipalidades de Chiclayo
}

\author{
Max Fernando Urbina Cárdenas * \\ Roger Fernando Chanduví Calderón ** \\ José Félix Zuloeta Salazar *** \\ Edgard Chapoñan Ramírez **** \\ Eveling Sussety Balcázar Paiva ****
}

RESUMEN

El presente estudio tuvo como propósito establecer el nivel de relación existente entre presupuesto participativo por resultados y toma de decisiones en la asignación y ejecución de proyectos de las municipalidades de la provincia de Chiclayo, 2019. La investigación fue descriptiva correlacional. La muestra estuvo conformada por los pobladores de cuatro municipalidades distritales más significativas de la provincia de Chiclayo: Chiclayo, La Victoria, José Leonardo Ortiz y Pimentel. La técnica empleada para la recolección de la información fue la encuesta; y el instrumento, el cuestionario para la evaluación del presupuesto participativo y toma de decisiones. Se realizó un análisis descriptivo y un análisis inferencial, para probar la hipótesis de investigación, mediante la técnica estadística de correlación de Pearson. Los resultados encontrados indican que existe una correlación entre el presupuesto participativo y toma de decisiones. En conclusión, la toma de decisiones que se realice para la ejecución de las obras será eficiente, una vez que el presupuesto participativo es concebido y aplicado como un instrumento de política y de gestión eficiente, mediante el cual las autoridades regionales y locales, y las organizaciones de la población debidamente representadas, definen un conjunto de acciones debidamente planificadas, con la finalidad de orientar los recursos de dicho presupuesto.

PALABRAS CLAVE: Presupuesto de Estado; participación social; proyectos de desarrollo; gestión.

* Docente. Escuela de Contabilidad, Facultad de Ciencias Empresariales, Universidad Señor de Sipán S.A.C., Pimentel - Chiclayo, Perú. ORCID: https://orcid.org/0000-0002-0474-5590. E-mail: urbinamax@crece.uss.edu.pe

**Docente. Universidad Señor de Sipán S.A.C., Pimentel - Chiclayo, Perú. ORCID: http://orcid.org/00000001-7023-0280. E-mail: rchanduvi@crece.uss.edu.pe

***Docente. Escuela de Contabilidad, Facultad de Ciencias Empresariales, Universidad Señor de Sipán S.A.C., Pimentel - Chiclayo, Perú. ORCID: https://orcid.org/0000-0002-5437-8557. E-mail: JFELIXZS@crece.uss.edu.pe

****Docente. Escuela de Contabilidad, Facultad de Ciencias Empresariales, Universidad Señor de Sipán S.A.C., Pimentel - Chiclayo, Perú. ORCID: https://orcid.org/0000-0001-7098-5755. E-mail: edgardch@crece.uss.edu.pe

*****Docente. Escuela de Contabilidad, Facultad de Ciencias Empresariales, Universidad Señor de Sipán S.A.C., Pimentel - Chiclayo, Perú. ORCID: https://orcid.org/0000-0002-4188-0724. E-mail: bpaivaes@crece.uss.edu.pe 


\section{Participatory budgeting for results and decision-making in Chiclayo municipalities}

ABSTRACT

The purpose of this study was to establish the level of relationship between participatory budgeting by results and decision-making in the assignment and execution of projects of the municipalities of the province of Chiclayo, 2019. The research was descriptive and correlational. The sample was made up of the residents of four most significant district municipalities in the province of Chiclayo: Chiclayo, La Victoria, José Leonardo Ortiz and Pimentel. The technique used to collect the information was the survey; and the instrument, the questionnaire for the evaluation of the participatory budget and decision-making. Descriptive analysis and inferential analysis were carried out to test the research hypothesis, using Pearson's statistical correlation technique. The results found indicate that there is a correlation between participatory budgeting and decision making. In conclusion, the decision-making that is carried out for the execution of the works will be efficient, once the participatory budget is conceived and applied as an instrument of efficient policy and management, through which the regional and local authorities, and the Duly represented population organizations define a set of duly planned actions, in order to direct the resources of said budget.

KEYWORDS: state budget; social participation; development projects; management.

\section{Introducción}

El presupuesto público es una herramienta importante para que el Estado cumpla su función esencial de dotar de servicios a la población, a través de la administración pública. En Perú, a partir de la dación de la Ley marco de presupuesto participativo Nº 28056 (2003), se implementan mecanismos para una asignación con características de ser equitativa, racional, eficiente y transparente, que permitan robustecer las relaciones entre el Estado y la Sociedad Civil, otorgando facultades a los que dirigen las organizaciones de tal forma que intervengan directamente en priorizar la inversión pública (Quispe et al, 2021), y participen en mecanismos de control y fiscalización, supervisando que se cumplan con los compromisos asumidos, alentar que se rindan cuentas claras, promoviendo el fortalecimiento de capacidades.

Durante los últimos años, en varios países latinoamericanos se vienen implementando cambios medulares en los modelos de gestión, los cuales permiten orientar de mejor manera 
el desempeño de la administración pública, teniendo como fundamento los resultados; también el fortalecimiento de sistemas que permitan desarrollar acciones de seguimiento y evaluación, mejorando la calidad del gasto público, el mejor desenvolvimiento de las instituciones y el impacto de las políticas públicas (Córdova, 2000).

En la actualidad se privilegia la administración que permita comprometer a los ciudadanos para obtener excelentes resultados en la rendición de cuentas, en contraposición al mero control en la aplicación de normativas y procedimientos.

En México, Arellano (2011) refiere que, a causa de una improductiva interferencia política, uso y abuso de excesiva normatividad, nulo o casi nulo apoyo por la innovación y la poca capacidad para adaptación a los cambios, los gestores públicos han visto debilitado su trabajo. Debido a la ausencia de instrumentos sustanciales como indicadores que midan un real desempeño, evaluaciones de procesos y procedimientos, fondos concursables, programas de mejoramiento de gestión y balances, se ha convenido proponer integrar todos ellos en el proceso presupuestario (Guzmán, 2014).

En Perú, las iniciativas de gestión por resultados son muy incipientes, dispersas y con poca o nula coordinación entre las organizaciones que tienen que ver con la administración pública; ello pone en riesgo desarrollar acciones que apoyen la consistencia y coherencia necesaria de la gestión. Los Convenios de Gestión de la PCM, de Administración por Resultados del MEF, de Gestión del FONAFE y los Acuerdos de Gestión en el sector salud, se han convertido en iniciativas como instrumentos de gestión; pero, lamentablemente carecen de la articulación y coordinación que permitan la planificación y evaluación de la gestión pública. El contexto organizacional de la administración pública en el Perú, desfavorece la orientación en la gestión hacia resultados; así, entre otros elementos, se evidencia precariedad en las áreas de: planificación, evaluación de la gestión pública y rendición de cuentas públicas (Kontz, 2014).

Asimismo, se observa que tanto en las actividades de programación como en las de ejecución, existen serios desajustes acorde con la realidad; hay un evidente divorcio de los objetivos estratégicos de los planes de desarrollo concertados por el Estado y la Sociedad Civil; en la población, las deficiencias son más sentidas por la ausencia de resultados palpables. Si bien el Ministerio de Economía y Finanzas, considera un aspecto importante la cobertura lograda, también reconoce que hay muchos limitantes que han dado paso al 
incumplimiento en la ejecución de obras de gran envergadura a nivel nacional; a lo cual se debe agregar el incumplimiento de los acuerdos de funcionarios del estado, la ausencia de compromiso político y la nula priorización de la inversión.

Distintas investigaciones y análisis realizados dan cuenta que el presupuesto, tal como se desarrolla en la actualidad, tiene una tarea pendiente: lograr consolidarse como un adecuado instrumento en la gestión, que permita promover provisión importante de los bienes de orden público, para el logro de resultados que beneficien a la comunidad. Al respecto, existen limitaciones, entre estas: la débil articulación entre el planeamiento y el presupuesto; la normatividad abundante que genera rigidez en la gestión; el marcado énfasis en la gestión financiera; compromisos laborales que generan muchos problemas en las asignaciones de las partidas presupuestarias; deficiencia en la evaluación del desempeño que determine si se logran o no los objetivos previstos; toma de decisiones que no se sustentan en evaluaciones significativas (Secretaría de Gestión Pública. Presidencia del Consejo de Ministros del Perú, 2021). Los diferentes instrumentos se encuentran desarticulados al no contar con instancias de coordinación sistemática; es por ello que aspectos como evaluación y planificación, siempre aparecen en niveles muy por debajo de los promedios regionales.

En Perú, las municipalidades distritales -como órganos de gobierno local- se rigen por presupuestos participativos que se brindan de manera anual. Sin embargo, al momento de invertir, los gobiernos locales priorizan el cuánto se destina en actividades y proyectos que se van a ejecutar, pero no se enfocan en la inversión de actividades que beneficien a poblaciones específicas, como por ejemplo las vulnerables. Los recursos, por tanto, no son utilizados de manera racional.

Así mismo, el Ministerio de Economía y Finanzas, sin considerar criterios importantes de evaluación sobre los resultados que beneficien a la población, solamente asigna recursos presupuestales para el desarrollo de programas sociales, como por ejemplo: el programa del vaso de leche, comedores del PRONAA, Programa de Alimentos Nutricionales, sin que se generen los resultados esperados.

Diversas investigaciones concluyen que la participación de los ciudadanos en la conducción de sus comunidades, por ende, de su bienestar, se ha convertido un componente determinante para las administraciones públicas de los países (Del Giorgio \& Girotto, 2020) En Chile se ha verificado que el éxito de las diversas políticas públicas tiene que ver con la 
inclusión de los ciudadanos en la gestión gubernamental (Pinochet, 2017). En Colombia, Quintero (2013) concluye que el conocimiento previo para implementar el presupuesto participativo evita las dificultades técnicas y complejas para su ejecución. La generación de estudios sobre participación, destaca los efectos positivos de los llamados casos paradigmáticos y la duda de replicación en casos emblemáticos no resueltos en Porto Alegro y Belo Horizonte (Vallejos, 2014).

En el marco del empoderamiento del ciudadano para supervisar, vigilar y verificar las acciones de la gestión pública, la Contraloría General de la República peruana promueve con mayor dinámica su participación (Meza, 2018). Así también, Bringas (2014) encontró que el presupuesto participativo influye de manera directa en la eficiencia y eficacia del manejo de los recursos públicos municipales, ya que su aplicación debe efectuarse en el marco de un diagnóstico que facilite la evaluación de la problemática de cada sector, apoyando de esta manera a priorizar las necesidades de la población, permitiendo identificar adecuadamente la cantidad y el uso apropiado de los recursos necesarios para obtener los resultados, de tal manera que se cumplan los objetivos planeados. Anampa (2018) asiente que el proceso participativo presupuestal ha contribuido para que las comunidades tengan una cultura participativa, ejerciendo acciones que aportan al impulso de la gobernabilidad.

El presupuesto participativo es un mecanismo que asigna de manera equitativa, racional, eficiente, eficaz y transparente los recursos públicos, que fortalece las relaciones Estado - Sociedad civil (Ley 28056, 2003). El presupuesto participativo es considerado por Ruiz, (2016) como "un mecanismo que se promueve para que la comunidad participe de manera directa en la toma de decisiones, y así involucrarla en la gestión del desarrollo de su comunidad, contribuyendo, además con fortalecer su cultura democrática, partiendo de una relación basada en la transparencia entre los actores comprometidos en el proceso". Barbarán y Rioja (2019), sostienen que "es una técnica abierta hacia determinados sectores de la administración pública o de la población beneficiaria, estableciéndose acuerdos en cuanto a la aplicación de los recursos y plazos establecidos". Es un mecanismo de participación social que permite, desde los habitantes de una comunidad hasta una nación, a organizarse con un presupuesto propio que permita conocer los ingresos y los gastos, decidiendo sobre dicho presupuesto, convirtiéndose en ente de evaluación y control. También se define como un 
sistema de formulación del presupuesto público, como un acto democrático de administrar las urbes, como proceso político que le da poder de decisión al ciudadano.

En el Perú, la Ley Marco de Presupuesto Participativo Nº 28056 (2003) establece los principios rectores del presupuesto participativo, a saber: participación, transparencia, igualdad, tolerancia, eficacia y eficiencia, equidad, competitividad, respeto a los acuerdos; también define las instancias de participación, las fases del proceso participativo, la oficialización de los compromisos y los mecanismos de vigilancia participativa. La misma ley de presupuesto participativo resalta la importancia de capacitar a los agentes que participan de las instancias del presupuesto participativo.

Cohaila (2009) afirma que es importante poder identificar a los principales actores del presupuesto participativo y determinar la función que les compete a cada uno: consejo de coordinación regional, local, municipal; los representantes de la sociedad civil y de las entidades del gobierno nacional; el equipo técnico que participa con voz, pero no con voto.

El tomar decisiones en la administración equivale principalmente a la resolución de inconvenientes en el mando empresarial. Los diagnósticos de problemas, las búsquedas y las evaluaciones de alternativas y la elección final de una decisión, constituyen las etapas básicas en el proceso de toma de decisiones y resolución de problemas. La toma de decisiones es considerada la elección que se hace entre varias alternativas (León, citado por Gutiérrez, 2014), la identificación y resolución de problemas que se presentan en todas las organizaciones (Le Moigne, citado por Gutiérrez 2014).

Las decisiones no son iguales, ni producen las mismas consecuencias, ni tampoco su adopción es de idéntica relevancia; por ello, Rodríguez, (2017) afirma que existen distintos tipos de decisiones. Para su clasificación destacaremos las más representativas: decisiones estratégicas dadas por los altos directivos; tácticas o de pilotaje tomadas por directivos intermedios; y operativas adoptadas por ejecutivos de nivel más inferior.

En este sentido, el presente artículo aborda el estudio del siguiente problema: ¿De qué manera el Presupuesto Participativo por Resultados se relaciona en la toma de decisiones de asignación y ejecución de proyectos de las municipalidades distritales de la provincia de Chiclayo, 2019? Se pretende, en cuanto a objetivo general: establecer el nivel de relación existente entre presupuesto participativo por resultados y toma de decisiones en la asignación y ejecución de proyectos de las municipalidades de la provincia de Chiclayo, 2019. 
Este objetivo contiene tres objetivos específicos: Identificar las características emergentes que tiene la toma de decisiones en la asignación y ejecución de los recursos públicos a través de proyectos de las municipalidades; identificar los factores influyentes en la relación entre presupuesto participativo por resultados y toma de decisiones; y determinar la existencia de una relación significativa entre presupuesto participativo por resultados y la toma de decisiones de la asignación y ejecución de proyectos.

\section{Material y método}

La investigación fue de tipo descriptivo correlacional, y diseño no experimental. La población estuvo constituida por los pobladores (representantes y población de los diferentes grupos sociales territoriales, organizaciones de la sociedad civil) de las municipalidades distritales de la Provincia de Chiclayo, la cual posee 20 distritos. Para la selección de los distritos, se aplicó el muestreo no probabilístico intencional, seleccionándose cuatro municipalidades distritales, las más representativas de la Provincia de Chiclayo, que son: Chiclayo, La Victoria, José Leonardo Ortiz y Pimentel. Para seleccionar el total de la muestra en los cuatro distritos, se aplicó el muestreo probabilístico estratificado, y para la obtención de la muestra por cada distrito, se empleó el muestreo aleatorio simple (MAS), quedando conformada cada muestra por distrito de la siguiente manera: Chiclayo: 305 pobladores; José Leonardo Ortiz: 183 pobladores; La Victoria: 84 pobladores; Pimentel: 30 pobladores; haciendo un total de 602 pobladores.

La encuesta fue la técnica empleada para la recolección de datos para ambas variables. El instrumento fue el cuestionario. El cuestionario de la variable presupuesto participativo, estuvo constituida por 18 preguntas; y el cuestionario toma de decisiones, 12 preguntas. Las preguntas para ambos cuestionarios, fueron de tipo cerrada, con respuesta (alternativa) en escala Likert, con 5 categorías: muy en desacuerdo = MD; en desacuerdo =ED; ni de acuerdo, ni en desacuerdo=NAND; de acuerdo = DA; muy de acuerdo = MA. Los cuestionarios fueron validados por juicio de expertos en el tema de estudio, teniendo en cuenta el marco teórico, y la operacionalización de las variables (dimensiones e indicadores).

Para el análisis de datos se empleó el software Excel y SPSS. Se realizó un análisis descriptivo, consistente en la elaboración de tablas y gráficos de distribución de frecuencias de doble entrada (tablas de contingencia) y estadísticas de tendencia central y de dispersión. El análisis inferencial se realizó mediante la prueba de Correlación de Pearson, la cual evalúa 
el grado de relación o interdependencia entre variables cuantitativas. Esta prueba se realizó para probar la hipótesis de investigación.

\section{Resultados}

\subsection{Análisis descriptivo del presupuesto participativo}

Con la finalidad de realizar un análisis descriptivo de la variable presupuesto participativo, se presenta un conjunto de tablas y gráficos de distribución de frecuencias de doble entrada (tablas de contingencia), por cada dimensión de esta variable: dimensión financiera, participativa, normativa y jurídica legal, y territorial. Cada tabla y gráfico (figura) clasifica a los pobladores, según municipalidad distrital y nivel de opinión (escala de Lickert).

\section{1.l. Dimensión financiera}

Tabla 1. Distribución adecuada de los recursos en su localidad.

\begin{tabular}{lccccc}
\hline \multirow{2}{*}{ Escala } & Chiclayo & $\begin{array}{c}\text { José } \\
\text { Leonardo } \\
\text { Ortiz }\end{array}$ & $\begin{array}{c}\text { La } \\
\text { Victoria }\end{array}$ & Pimentel & Total \\
\hline Muy en desacuerdo & 0 & 11 & 2 & 0 & 13 \\
En desacuerdo & $0,0 \%$ & $1,8 \%$ & $0,3 \%$ & $0,0 \%$ & $2,2 \%$ \\
& 139 & 70 & 21 & 11 & 241 \\
Ni de acuerdo, ni en & $23,1 \%$ & $11,6 \%$ & $3,5 \%$ & $1,8 \%$ & $40,0 \%$ \\
desacuerdo & 22 & 55 & 32 & 1 & 110 \\
De acuerdo & $3,7 \%$ & $9,1 \%$ & $5,3 \%$ & $0,2 \%$ & $18,3 \%$ \\
& 144 & 44 & 28 & 18 & 234 \\
Muy de acuerdo & $23,9 \%$ & $7,3 \%$ & $4,7 \%$ & $3,0 \%$ & $38,9 \%$ \\
& 0 & 3 & 1 & 0 & 4 \\
\multicolumn{1}{c}{ Total } & $0,0 \%$ & $0,5 \%$ & $0,2 \%$ & $0,0 \%$ & $0,7 \%$ \\
\hline & 305 & 183 & 84 & 30 & 602 \\
& $50,7 \%$ & $30,4 \%$ & $14,0 \%$ & $5,0 \%$ & $100,0 \%$ \\
\hline
\end{tabular}

Fuente: Base de datos del cuestionario de presupuesto participativo.

Con respecto a que qué tan de acuerdo están con los recursos asignados en el Presupuesto Participativo (tabla 1), se observa que un 40,0\% de los contribuyentes de los cuatro distritos afirman estar en desacuerdo con tal asignación, mientras un 38,9\% afirman estar de acuerdo. 
REVISTA DE LA UNIVERSIDAD DEL ZULIA. $3^{a}$ época. Año $12 \mathrm{~N}^{\circ} 34,2021$

M. F. Urbina Cárdenas et al. /// Presupuesto participativo por resultados y la toma decisiones ... 185-205

DOI: http://dx.doi.org/10.46925//rdluz.34.12

\subsubsection{Dimensión participativa}

Tabla 2. Pertinencia adecuada de la participación ciudadana en el Presupuesto Participativo.

\begin{tabular}{lccccc}
\hline \multicolumn{1}{c}{ Escala } & Chiclayo & $\begin{array}{c}\text { José } \\
\text { Leonardo } \\
\text { Ortiz }\end{array}$ & $\begin{array}{c}\text { La } \\
\text { Victoria }\end{array}$ & Pimentel & Total \\
\hline Muy en desacuerdo & 8 & 6 & 0 & 0 & 14 \\
En desacuerdo & $1,3 \%$ & $1,0 \%$ & $0,0 \%$ & $0,0 \%$ & $2,3 \%$ \\
Ni de acuerdo, ni en desacuerdo & 69 & 10 & 4 & 2 & 85 \\
& $11,5 \%$ & $1,7 \%$ & $0,7 \%$ & $0,3 \%$ & $14,1 \%$ \\
De acuerdo & 66 & 32 & 3 & 0 & 101 \\
& $11,0 \%$ & $5,3 \%$ & $0,5 \%$ & $0,0 \%$ & $16,8 \%$ \\
Muy de acuerdo & 162 & 122 & 71 & 28 & 383 \\
& $26,9 \%$ & $20,3 \%$ & $11,8 \%$ & $4,7 \%$ & $63,6 \%$ \\
& 0 & 13 & 6 & 0 & 19 \\
& $0,0 \%$ & $2,2 \%$ & $1,0 \%$ & $0,0 \%$ & $3,2 \%$ \\
\hline \multirow{2}{*}{ Total } & 305 & 183 & 84 & 30 & 602 \\
& $50,7 \%$ & $30,4 \%$ & $14,0 \%$ & $5,0 \%$ & $100,0 \%$ \\
\hline
\end{tabular}

Fuente: Base de datos del cuestionario de presupuesto participativo.

En cuanto a si se considera pertinente la participación ciudadana en el Presupuesto Participativo (tabla 2), un 63,6\% del total de contribuyentes afirma estar de acuerdo con que exista una participación por parte de la ciudadanía; en cambio un 14,1\% afirma estar en desacuerdo con dicha actividad.

\subsubsection{Dimensión normativa y jurídica legal}

Tabla 3. El proceso de formalización y legalización del Presupuesto Participativo en la localidad cumple con la normativa legal establecida.

\begin{tabular}{lccccc}
\hline \multirow{2}{*}{ Escala } & Chiclayo & $\begin{array}{c}\text { José } \\
\text { Leonardo } \\
\text { Ortiz }\end{array}$ & $\begin{array}{c}\text { La } \\
\text { Victoria }\end{array}$ & Pimentel & Total \\
\hline Muy en desacuerdo & 6 & 34 & 6 & 0 & 46 \\
En desacuerdo & $1,0 \%$ & $5,6 \%$ & $1,0 \%$ & $0,0 \%$ & $7,6 \%$ \\
Ni de acuerdo, ni en desacuerdo & 77 & 59 & 54 & 29 & 219 \\
& $12,8 \%$ & $9,8 \%$ & $9,0 \%$ & $4,8 \%$ & $36,4 \%$ \\
De acuerdo & 49 & 61 & 18 & 1 & 129 \\
& $8,1 \%$ & $10,1 \%$ & $3,0 \%$ & $0,2 \%$ & $21,4 \%$ \\
Muy de acuerdo & 173 & 25 & 6 & 0 & 204 \\
& $28,7 \%$ & $4,2 \%$ & $1,0 \%$ & $0,0 \%$ & $33,9 \%$ \\
& 0 & 4 & 0 & 0 & 4 \\
& $0,0 \%$ & $0,7 \%$ & $0,0 \%$ & $0,0 \%$ & $0,7 \%$ \\
\hline \multirow{2}{*}{ Total } & 305 & 183 & 84 & 30 & 602 \\
& $50,7 \%$ & $30,4 \%$ & $14,0 \%$ & $5,0 \%$ & $100,0 \%$ \\
\hline
\end{tabular}

Fuente: Base de datos del cuestionario de presupuesto participativo. 
En lo referido a si el proceso de formalización y legalización del Presupuesto Participativo en la localidad cumple con la normativa legal establecida (tabla 3), un 36,4\% del total de contribuyentes encuestados afirmaron estar en desacuerdo, un 21,4\% no estuvieron de acuerdo, ni en desacuerdo; en cambio un 33,9\% afirmaron estar de acuerdo con dicho proceso.

\subsubsection{Dimensión territorial}

Tabla 4. Existencia de descentralización en la asignación del Presupuesto Participativo

\begin{tabular}{lccccc}
\hline \multirow{2}{*}{ Escala } & Chiclayo & $\begin{array}{c}\text { José } \\
\text { Leonardo } \\
\text { Ortiz }\end{array}$ & $\begin{array}{c}\text { La } \\
\text { Victoria }\end{array}$ & Pimentel & Total \\
& 0 & 14 & 3 & 0 & 17 \\
Muy en desacuerdo & $0,0 \%$ & $2,3 \%$ & $0,5 \%$ & $0,0 \%$ & $2,8 \%$ \\
En desacuerdo & 214 & 40 & 43 & 24 & 321 \\
& $35,5 \%$ & $6,6 \%$ & $7,1 \%$ & $4,0 \%$ & $53,3 \%$ \\
Ni de acuerdo, ni en desacuerdo & 74 & 103 & 23 & 6 & 206 \\
& $12,3 \%$ & $17,1 \%$ & $3,8 \%$ & $1,0 \%$ & $34,2 \%$ \\
De acuerdo & 14 & 26 & 15 & 0 & 55 \\
& $2,3 \%$ & $4,3 \%$ & $2,5 \%$ & $0,0 \%$ & $9,1 \%$ \\
Muy de acuerdo & 3 & 0 & 0 & 0 & 3 \\
& $0,5 \%$ & $0,0 \%$ & $0,0 \%$ & $0,0 \%$ & $0,5 \%$ \\
\hline \multirow{2}{*}{ Total } & 305 & 183 & 84 & 30 & 602 \\
& $50,7 \%$ & $30,4 \%$ & $14,0 \%$ & $5,0 \%$ & $100,0 \%$ \\
\hline
\end{tabular}

Fuente: Base de datos del cuestionario de presupuesto participativo.

En lo que se refiere al proceso de descentralización en la asignación del Presupuesto Participativo (tabla 4), un 53,3\% del total de encuestados afirmaron encontrarse en desacuerdo con este proceso, en cambio un 9,1\% afirmaron estar de acuerdo. Cabe señalar que un $34,2 \%$ de los encuestados no estuvieron de acuerdo, ni en desacuerdo con dicho proceso.

\subsection{Análisis descriptivo de toma de decisiones}

Para realizar el análisis descriptivo de la variable toma de decisiones, al igual que el análisis de la variable presupuesto participativo, se elaboraron tablas y gráficos de distribución de frecuencias de doble entrada. 
Tabla 5. Forma como participa la ciudadana en la toma de decisiones del presupuesto participativo.

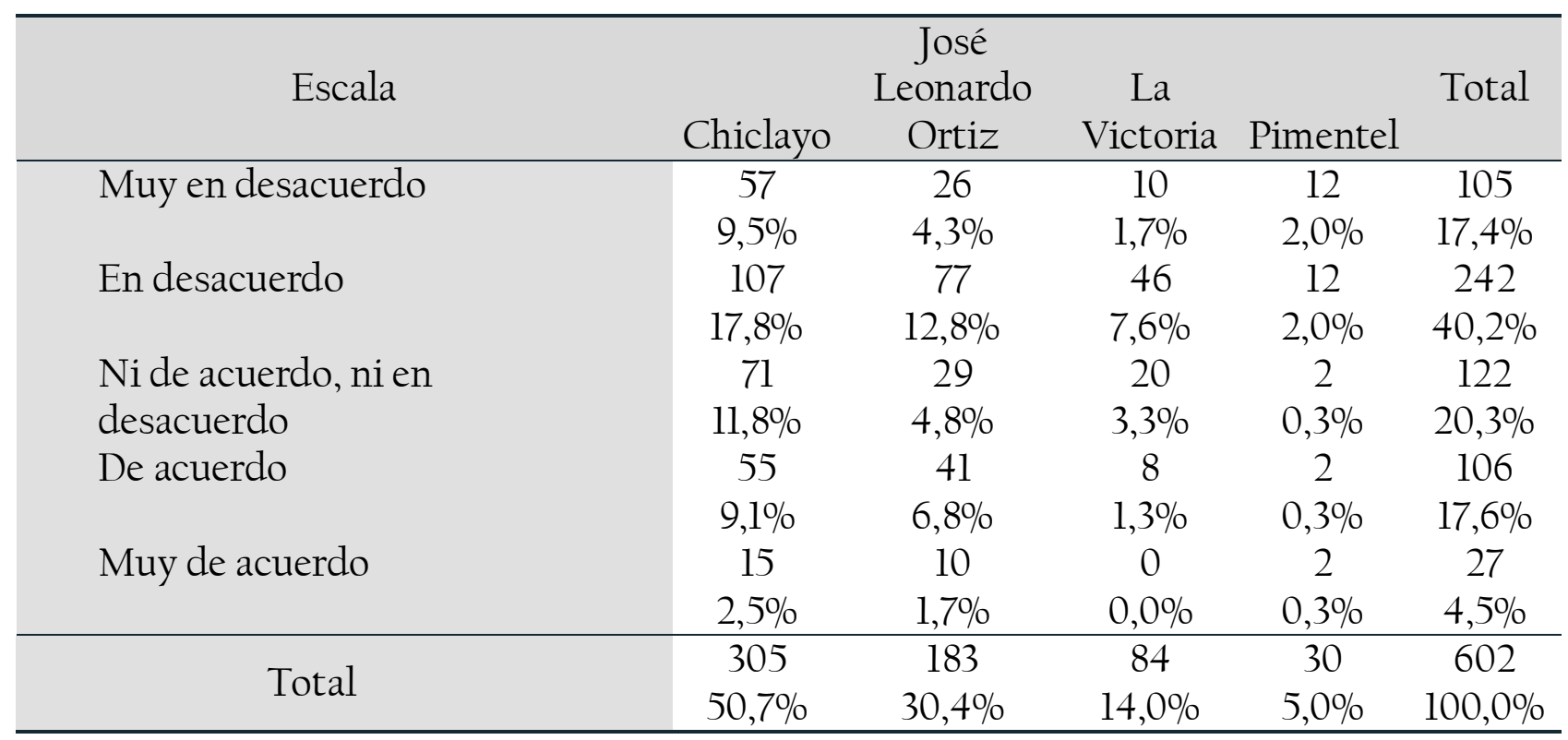

Fuente: Base de datos del cuestionario de toma de decisiones.

Con respecto a la forma de participación ciudadana en la toma de decisiones (tabla 5), se observa que un 40,2\% de los encuestados en los 4 distritos, afirman estar en desacuerdo con dicha participación; un 17,4\% afirma estar muy en desacuerdo. En cambio, un 17,6\% y 4,5\% opina respectivamente, estar de acuerdo y muy de acuerdo.

Tabla 6. El alcalde y los funcionarios de la municipalidad, toman decisiones teniendo en cuenta la consulta de los agentes participantes.

\begin{tabular}{lccccc}
\hline \multirow{2}{*}{ Escala } & \multicolumn{5}{c}{ José } \\
& Chiclayo & Ortiz & & Tato \\
& Oictoria & Pimentel & \\
\hline Muy en desacuerdo & 32 & 51 & 12 & 12 & 107 \\
& $5,3 \%$ & $8,5 \%$ & $2,0 \%$ & $2,0 \%$ & $17,8 \%$ \\
En desacuerdo & 171 & 93 & 57 & 13 & 334 \\
& $28,4 \%$ & $15,4 \%$ & $9,5 \%$ & $2,2 \%$ & $55,5 \%$ \\
Ni de acuerdo, ni en & 53 & 21 & 8 & 0 & 82 \\
desacuerdo & $8,8 \%$ & $3,5 \%$ & $1,3 \%$ & $0,0 \%$ & $13,6 \%$ \\
De acuerdo & 37 & 15 & 5 & 4 & 61 \\
& $6,1 \%$ & $2,5 \%$ & $0,8 \%$ & $0,7 \%$ & $10,1 \%$ \\
Muy de acuerdo & 12 & 3 & 2 & 1 & 18 \\
& $2,0 \%$ & $0,5 \%$ & $0,3 \%$ & $0,2 \%$ & $3,0 \%$ \\
\hline \multirow{2}{*}{ Total } & 305 & 183 & 84 & 30 & 602 \\
& $50,7 \%$ & $30,4 \%$ & $14,0 \%$ & $5,0 \%$ & $100,0 \%$ \\
\hline
\end{tabular}

Fuente: Base de datos del cuestionario de toma de decisiones. 
En la tabla 6 se observa la consulta a los encuestados, para conocer si consideran que el alcalde y los funcionarios de la municipalidad toman decisiones teniendo en cuenta la consulta de los agentes participantes: un 55,5\% de los encuestados afirmaron estar en desacuerdo, y un 17,8\% muy en desacuerdo con dicha actividad. En cambio, un 10,1\% y un 3,0\% opinaron respectivamente, estar de acuerdo y muy de acuerdo.

Tabla 7. El presupuesto participativo es base para una buena toma de decisiones municipales.

\begin{tabular}{|c|c|c|c|c|c|}
\hline Escala & Chiclayo & $\begin{array}{c}\text { José } \\
\text { Leonardo } \\
\text { Ortiz }\end{array}$ & $\begin{array}{c}\mathrm{La} \\
\text { Victoria }\end{array}$ & Pimentel & Total \\
\hline \multirow[t]{2}{*}{ Muy en desacuerdo } & 56 & 44 & 15 & 11 & 126 \\
\hline & $9,3 \%$ & $7,3 \%$ & $2,5 \%$ & $1,8 \%$ & $20,9 \%$ \\
\hline \multirow[t]{2}{*}{ En desacuerdo } & 172 & 111 & 64 & 16 & 363 \\
\hline & $28,6 \%$ & $18,4 \%$ & $10,6 \%$ & $2,7 \%$ & $60,3 \%$ \\
\hline Ni de acuerdo, ni en & 43 & 14 & 4 & 2 & 63 \\
\hline desacuerdo & $7,1 \%$ & $2,3 \%$ & $0,7 \%$ & $0,3 \%$ & $10,5 \%$ \\
\hline \multirow[t]{2}{*}{ De acuerdo } & 29 & 10 & 1 & 1 & 41 \\
\hline & $4,8 \%$ & $1,7 \%$ & $0,2 \%$ & $0,2 \%$ & $6,8 \%$ \\
\hline \multirow[t]{2}{*}{ Muy de acuerdo } & 5 & 4 & 0 & 0 & 9 \\
\hline & $0,8 \%$ & $0,7 \%$ & $0,0 \%$ & $0,0 \%$ & $1,5 \%$ \\
\hline \multirow[t]{2}{*}{ Total } & 305 & 183 & 84 & 30 & 602 \\
\hline & $50,7 \%$ & $30,4 \%$ & $14,0 \%$ & $5,0 \%$ & $100,0 \%$ \\
\hline
\end{tabular}

Fuente: Base de datos del cuestionario de toma de decisiones.

En lo que respecta a si se considera que el presupuesto participativo es base para una buena toma de decisiones municipales, se observa en la tabla 7 que del total de encuestados, un $60,3 \%$ y un $20,9 \%$ de los encuestados afirmaron, respectivamente, estar en desacuerdo y muy en desacuerdo con esta manera de tomar decisiones.

\subsection{Presentación de estadísticas descriptivas}

Este análisis corresponde también al análisis descriptivo de las variables en estudio, y tiene como finalidad describir a través de diferentes indicadores estadísticos (medidas estadísticas de tendencia central y dispersión) las variables presupuesto participativo y toma de decisiones. 
Tabla 8. Estadísticas descriptivas de la evaluación del presupuesto participativo y toma de decisiones. Distritos de Chiclayo, José Leonardo Ortiz, La Victoria y Pimentel, 2019.

\begin{tabular}{|c|c|c|c|}
\hline \multicolumn{2}{|c|}{ Estadísticas } & Presupuesto participativo & Toma de decisiones \\
\hline \multirow[t]{2}{*}{$\mathrm{N}$} & Válido & 602 & 602 \\
\hline & Perdidos & 0 & 0 \\
\hline \multicolumn{2}{|c|}{ Media } & 51,17 & 35,47 \\
\hline \multicolumn{2}{|c|}{ Mediana } & 51,00 & 36,00 \\
\hline \multicolumn{2}{|c|}{ Moda } & 49 & 34 \\
\hline \multicolumn{2}{|c|}{ Desviación estándar } & 5,316 & 2,847 \\
\hline \multicolumn{2}{|c|}{ Mínimo } & 30 & 26 \\
\hline \multicolumn{2}{|c|}{ Máximo } & 69 & 45 \\
\hline
\end{tabular}

Fuente: Base de datos de los cuestionarios de presupuesto participativo y toma de decisiones.

En la tabla 8 se aprecian las estadísticas descriptivas (medidas) de tendencia central y dispersión, en la que se observa en cuanto al presupuesto participativo que el puntaje mínimo y máximo en la evaluación de esta variable, por parte de los encuestados, fue 30 y 69 puntos; siendo el puntaje promedio 51,17 puntos. El valor de la mediana nos indica que el 50\% de los encuestados, calificaron al presupuesto participativo en menos de 51,00 puntos. La moda, nos indica que el puntaje más frecuente en la evaluación del presupuesto participativo fue 49 puntos. La medida de dispersión, desviación estándar, nos indica que los puntajes en la evaluación del presupuesto participativo se desvían en 5,316 puntos del puntaje promedio.

En lo referente a toma de decisiones, el puntaje mínimo y máximo, en la evaluación de esta variable, fue 26 y 45 puntos, siendo el puntaje promedio 35,47. El valor de la mediana, nos indica que el 50\% de los encuestados calificaron a la toma de decisiones en menos de 36,00. El valor modal, nos indica que el puntaje más frecuente en la evaluación de la toma de toma de decisiones fue 34. La desviación estándar muestra que los puntajes en la evaluación de la toma de decisiones se desvían en 2,847 de su puntaje promedio.

\subsection{Análisis de correlación}

Este procedimiento estadístico se realizó con el propósito de responder a la pregunta de investigación y demostrar la hipótesis de estudio. En primer lugar, se elaboró el diagrama de dispersión con la finalidad de observar el comportamiento de los datos (puntos) y verificar el tipo de relación que existe entre las variables. También, se presenta la prueba de hipótesis para la correlación, mediante la técnica estadística de Pearson. 
Figura 1. Diagrama de dispersión

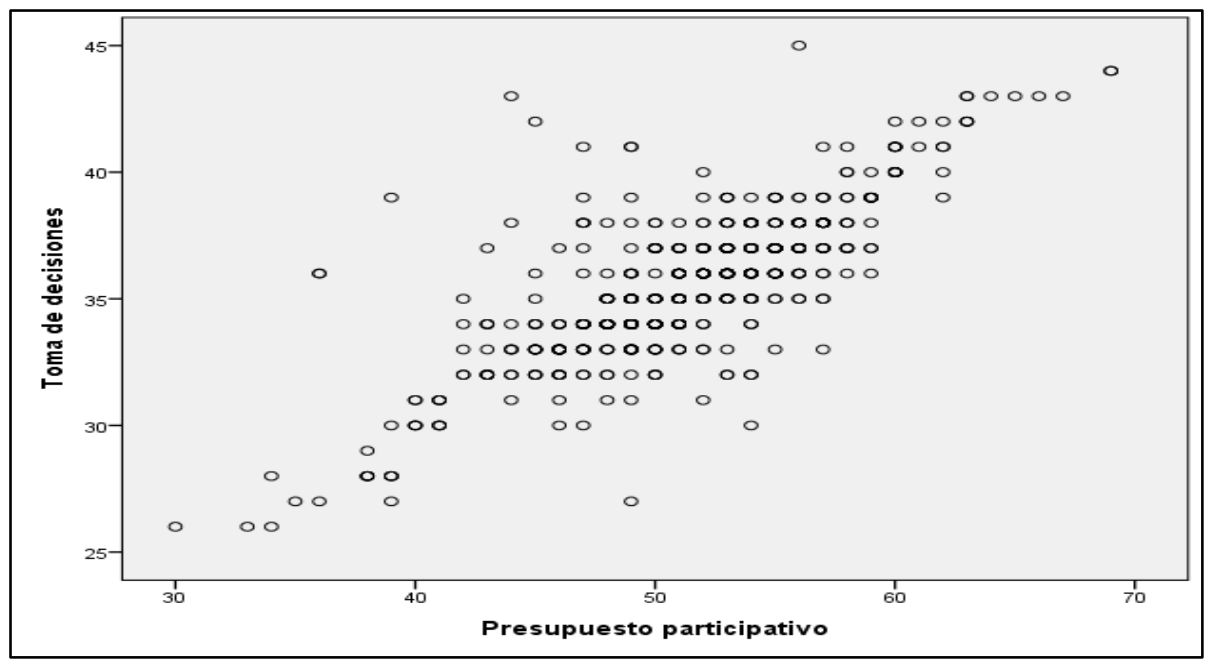

El diagrama de dispersión (nube de puntos, Figura 1), permite observar que a medida que aumentan los puntajes en la evaluación del presupuesto participativo, los puntajes de toma de decisiones también aumentan; evidenciando una correlación significativa positiva.

\subsection{Prueba de hipótesis para la correlación}

Esta prueba se realizó con la finalidad de probar la hipótesis de investigación: Existe una relación significativa entre presupuesto participativo por resultados y la toma de decisiones en la asignación y ejecución de proyectos.

Procedimiento:

1. Planteamiento de hipótesis estadísticas:

$\mathrm{H}_{0}: \rho=0$ (No existe asociación significativa entre presupuesto participativo y toma de decisiones)

$\mathrm{H}_{1}: \rho \neq 1$ (Existe asociación significativa entre presupuesto participativo y toma de decisiones)

2. Nivel de significancia: $\alpha=0,05$

3. Regla de decisión: Si p-valor $\geq \alpha \rightarrow$ Se acepta $\mathrm{H}_{0}$

Si p-valor $<\alpha \rightarrow$ Se $\mathrm{H}_{0}$ se rechaza 
REVISTA DE LA UNIVERSIDAD DEL ZULIA. $3^{\text {a }}$ época. Año 12 N 34, 2021

M. F. Urbina Cárdenas et al. /// Presupuesto participativo por resultados y la toma decisiones ... 185-205 DOI: http://dx.doi.org/10.46925//rdluz.34.12

Tabla 9. Prueba de correlación de Pearson.

\begin{tabular}{llcc}
\hline Variables & & $\begin{array}{c}\text { Presupuesto } \\
\text { participativo }\end{array}$ & $\begin{array}{c}\text { Toma de } \\
\text { decisiones }\end{array}$ \\
\hline Presupuesto participativo & Correlación de Pearson & 1 &, $783^{* *}$ \\
& Sig. (bilateral) & 602 & 600 \\
\multirow{3}{*}{ Toma de decisiones } & $\mathrm{N}$ &, $783^{* *}$ & 602 \\
& Correlación de Pearson &, 000 & 1 \\
& Sig. (bilateral) & 602 & 602 \\
\hline
\end{tabular}

F**. Correlación significativa en el nivel 0,0l (bilateral).

En la tabla 9 se presenta el análisis de correlación entre el presupuesto participativo y toma de decisiones, en la que se observa que el p-valor (Sig. (Bilateral)) =0,000 es menor al nivel de significación $(\alpha=0,05)$; por lo tanto, de acuerdo a la regla de decisión, se rechaza la hipótesis nula $\left(\mathrm{H}_{0}\right)$ y se concluye que existe correlación significativa fuerte y directa $(\mathrm{r}=$ $0,783^{* *}$ ) entre presupuesto participativo y toma de decisiones.

\section{Discusión}

El propósito del estudio consistió en determinar de qué manera el presupuesto participativo por resultados se relaciona con la toma de decisiones en las municipalidades de la provincia de Chiclayo, 2019. Para el logro de dicho objetivo, se realizó un estudio descriptivo para analizar la gestión del presupuesto participativo y la toma de decisiones por los funcionarios municipales y la población involucrada. De igual manera, se realizó un estudio inferencial consistente en determinar el nivel de relación entre las variables en estudio, a través de la prueba de correlación de Pearson.

El análisis descriptivo del presupuesto participativo evidenció deficiencias en el proceso de planificación y gestión, en lo que respecta a la dimensión financiera, participativa, normativa y jurídica legal, así como la territorial. Estudios realizados en la Municipalidad de Independencia, corroboran estos resultados, encontrándose que la gestión del presupuesto por resultado estaba en un nivel regular con $75.5 \%$ (Córdova, 2020); igualmente en la Municipalidad Distrital de Querecotillo, donde la gestión del presupuesto participativo fue calificada como regular (55\%) (Reyes, 2020). La falta de conocimiento y manejo de los diferentes procesos de gestión de los actores del proceso del presupuesto participativo, dificulta el logro de los objetivos o metas propuestas en los proyectos. En el caso de la 
participación civil, la problemática se presenta por la falta de una adecuada organización y priorización de intereses en función de resultados del colectivo, teniendo en cuenta los objetivos del Plan de Desarrollo Concertado (Bravo, 2019).

En el transcurso de la investigación, se identificaron las dificultades para realizar una buena gestión del presupuesto participativo: la baja eficiencia en la asignación y ejecución de los recursos públicos, de acuerdo a las prioridades que se consideran en el plan de desarrollo concertado. La falta de participación de los representantes de la organización civil, no fortalece la relación entre la Municipalidad y las instituciones civiles; no se establecen formas democráticas en los procesos de gestión a desarrollar, para cumplir con los objetivos estratégicos del plan de desarrollo concertado. Esta nula o escasa participación ciudadana representa un factor determinante para las administraciones públicas, dado que la participación de los ciudadanos en la gestión gubernamental asegura el éxito de las diversas políticas públicas. Sin embargo, se ha evidenciado en los últimos años la poca participación ciudadana en la gestión pública local del país (Pinochet, 2017). Las políticas nacionales de Presupuesto Participativo no han tenido un éxito generalizado, que promueva la participación ciudadana a nivel local, la transparencia fiscal, ni gobiernos municipales eficientes (Goldfrank, 2016). Un informe sobre Cultura Cívica en la Ciudad de México en 2017, refleja que un 64\% de los participantes manifestó desconocer para qué sirven los mecanismos de participación ciudadana (Comités Ciudadanos y Consejos de los Pueblos) (Larrosa y Bermúdez, 2019).

En lo que se refiere al análisis descriptivo de la toma de decisiones, los resultados revelan dificultades relacionadas con la falta de involucramiento y participación ciudadana, falta de criterios: técnicos, políticos y legales; lo que acarrea tomar decisiones bajo riesgo en la ejecución de las obras públicas. La falta de participación ciudadana no permite una adecuada formulación del Presupuesto Participativo, lo que origina una deficiente toma de decisiones en todos los procesos y procedimientos que se desarrollan en la planificación y ejecución de los proyectos. Esta situación problemática se corrobora con lo encontrado en el nivel de toma de decisiones en la municipalidad de Sullana, Piura, donde el grupo de estudio calificó la evaluación de toma de decisiones como medio (50\%); situación diferente se presentó en la Municipalidad Provincial de Requena, donde el proceso de toma de decisiones se viene desarrollando de manera adecuada, ya que los directivos poseen las suficientes 
competencias, controlando y analizando de manera regular las decisiones tomadas (Amias y Amasifuen, 2020).

Uno de los grandes factores asociados a una gestión eficiente, es la participación ciudadana relacionada con involucramiento en las diferentes acciones a desarrollar en el presupuesto participativo. Al ser consultada la población de los distritos muestreados, se pudo encontrar que un 63,6\% de la muestra afirmó estar de acuerdo con la participación ciudadana en el presupuesto participativo. Para el caso de la transparencia de la conformación del Consejo Participativo, un 66,8\% de la población afirmó estar en desacuerdo como se realiza; otro aspecto importante de los resultados encontrados es la falta de participación de las mujeres, donde un $72,3 \%$ de los ciudadanos encuestados afirmaron que estaban de acuerdo con dicha participación. Estos resultados se relacionan con el propósito de la Contraloría General de la República, institución que asume la participación ciudadana como mecanismo de vigilancia y denuncia, a fin de que se tomen decisiones de índole correctivos (Meza, 2018).

La participación ciudadana es el factor fundamental en una adecuada gestión del presupuesto participativo y la toma de decisiones. Los estudios realizados evidencian este aspecto, como lo realizado en México, donde se resalta el ejemplo de los presupuestos públicos participativos como elementos que contribuyen en la consolidación y mejora de la calidad de la democracia, pero cuyo impacto (tal y como lo revelan los ejercicios de Presupuesto Participativo en la Ciudad de México) es aún incipiente (Valverde, Gutiérrez, y García, 2013). La participación ciudadana se ha vuelto un factor determinante para las administraciones públicas, pues asegura el éxito de las diversas políticas públicas. Sin embargo, se ha evidenciado en los últimos años la poca participación ciudadana en la gestión pública local del país (Pinochet, 2017).

El análisis correlacional evidenció la existencia de un nivel de asociación positivo significativo entre las variables presupuesto participativo y toma de decisiones, con un coeficiente de correlación de $0,783^{* *}$. De igual manera, la prueba de hipótesis para la correlación lineal simple, confirmó que existe asociación significativa entre las variables consideradas en el estudio, ya que la significación bilateral $(0,000)$ resultó ser menor al nivel de significación 0,05. Estos resultados se corroboran con otro estudio, donde se demostró que el coeficiente de relación de Pearson indica una relación significativa fuerte entre la 
variable independiente y dependiente, lo que demuestra que el diseño de un plan estratégico socializado va a contribuir a una mejor toma de decisiones (Castañeda, 2021).

Otros estudios realizados confirman la relación entre las variables de estudio de esta investigación. En la Municipalidad de Independencia, los resultados inferenciales indican que existe relación positiva considerable entre presupuesto participativo y gestión por resultado, lo cual se demuestra con la significancia bilateral o valor equivalente a $0.00<0.05$ y el coeficiente de correlación de Spearman de 0.681 (Córdova, 2020). El estudio realizado en la Municipalidad de Querotillo, corrobora lo encontrado en el presente estudio, pues se demostró la existencia de una relación altamente significativa entre la participación ciudadana y la gestión del presupuesto participativo de la municipalidad, con un coeficiente de correlación de Pearson de 0,799, entre variables (Reyes, 2020). De la misma manera, el estudio realizado por Carbajal, determinó que existen evidencias suficientes para afirmar que el presupuesto participativo tiene relación positiva considerable $(\mathrm{Rho}=, 730)$ y significativa ( $\mathrm{p}$ valor $=0.000$ menor que 0.05 ) con la ejecución de obras en la provincia de Huaraz, 2018 (Carbajal, 2018).

\section{Conclusiones}

Se determinó que la toma de decisiones que se realice para la ejecución de las obras será eficiente, siempre y cuando el presupuesto participativo sea concebido y aplicado como un instrumento de política y de gestión eficiente, mediante el cual las autoridades locales y regionales, y la población debidamente organizada y representada, definan un conjunto de acciones debidamente planificadas, con el propósito de orientar los recursos de dicho presupuesto.

El análisis del aspecto financiero del presupuesto participativo, relacionado con la asignación y distribución de los recursos, el porcentaje del presupuesto municipal, la rendición de cuentas y la recaudación fiscal, evidenció que este proceso presenta grandes deficiencias en su ejecución en Chiclayo.

En lo que respecta a la participación ciudadana, se encontró deficiencias en el nivel participativo e involucramiento de las diferentes instituciones y conglomerados de la población (juntas y comités vecinales, clubes de madres, sindicatos, asociaciones de pueblos, jóvenes), en el proceso de planificación y gestión del presupuesto participativo. 
Un aspecto importante del presupuesto participativo es el que está relacionado con la dimensión territorial, ya que se presentan dificultades en la priorización de asignación del presupuesto para las zonas rurales, así como la forma de descentralización en la asignación de los recursos económicos para los sectores más empobrecidas de la población y zonas vulnerables.

El proceso de toma de decisiones de las diferentes administraciones municipales, relacionado con el presupuesto participativo, presenta deficiencias, ya que no tiene en cuenta la participación ciudadana, las prioridades de inversión de las obras públicas; no se tiene en cuenta la planificación, ejecución y evaluación presupuestal; así como tampoco la prioridad de las obras públicas.

\section{Referencias}

Amias, R., \& Amasifuen, K. (2020) La información contable y su relación con la toma de decisiones en la Municipalidad Provincial de Requena, Loreto año 2019 (tesis de pregrado) Universidad Nacional De La Amazonía Peruana. Recuperado de https://alicia.concytec.gob.pe/vufind/Record/UNAP_2c84ba2d6e2d3ea759353dc4f15d9722

Anampa, J. (2018). Análisis del presupuesto participativo de la municipalidad distrital de Los Olivos, periodo 20162017.2 Recuperado de http://repositorio.ucv.edu.pe/bitstream/handle/UCV/21369/Anampa_TJM.pdf?sequence=1\& isAllowed $=\mathrm{y}$

Arellano, D. (2009). Dilemas y potencialidades de los presupuestos orientados a resultados: presupuestos orientados a resultados: límites del gerencialismo en la reforma presupuestal. Recuperado http://www.top.org.ar/ecgp/FullText/000000/ARELLANO\%20GAULT,\%20David\%20 \%20Dilemas\%20y\%20potencialidades\%20de\%20los\%20presupuestos.pdf

Barbarán, O., \& Rioja, S. (2019). El proceso de presupuesto participativo y la satisfacción de los agentes participantes en la Municipalidad Provincial de Chiclayo. Recuperado de http://repositorio.unprg.edu.pe/bitstream/handle/UNPRG/8179/BC4561\%20ODAR\%20BA RBARAN-RIOJA\%20VALLE.pdf?sequence $=1 \&$ isAllowed $=y$

Bravo, C (2019). "Participación ciudadana y el presupuesto participativo en la Municipalidad Provincial de Huaytará - Huancavelica 2019 " (tesis posgrado). Universidad César Vallejo, Perú. Recuperado https://alicia.concytec.gob.pe/vufind/Record/UCVV_5ff927b227be0a85f2ca8a0cfbf267bf

Bringas, R. (2014). El presupuesto participativo y la calidad de la gestión de los recursos públicos en las municipalidades distritales de la región Ayacucho. Recuperado de http://www.repositorioacademico.usmp.edu.pe/bitstream/usmp/l107/3/bringas_dru.pdf 
Carbajal Y. (2018). Presupuesto participativo y la ejecución de obras públicas en la provincia de Huaraz, 2018. Tesis de posgrado. UCV. Recuperado de file:///C:/Users/usuario/Downloads/TESIS\%20POST\%20GRADO\%20\%20\%2OPRESUPUESTO\%2OPARTICIPATIVO.pdf

Castañeda, J (2021). Plan estratégico para la toma de decisiones en la municipalidad provincial de Chiclayo. Revista Científica Pakamuros, 9(1), 66-75. Recuperado de https://doi.org/10.37787/pakamuros-unj.v9il.164

Castañeda, T (2021). "Gestión de conflictos vecinales y toma de decisiones en la municipalidad de Sullana, Piura" (tesis posgrado). Universidad César Vallejo, Perú. Recuperado de https://repositorio.ucv.edu.pe/handle/20.500.12692/59962

Córdova, F. (2007). El presupuesto por resultados: un instrumento innovativo de gestión pública. Recuperado https:/www.cepal.org/ilpes/noticias/paginas/2/36342/presupuestoporresultados_F_CORD OVA.pdf

Córdova, G (2020). " Presupuesto participativo y gestión por resultados en la Municipalidad de Independencia" (tesis posgrado). Universidad César Vallejo, Perú. Recuperado de https://alicia.concytec.gob.pe/vufind/Record/UCVV_e4el89b094c293cld024498b396b925e

Del Giorgio Solfa, F.; Girotto, L.M. (2020). Municipal Development Forums: model for the improvement of local productive systems, Revista de Estudios Políticos y Estratégicos, 8 (2), 122 132. https://sitios.vtte.utem.cl/revistaepe/wp-content/uploads/sites/7/2021/0l/revistaestudios-politicos-estrategicos-epe-vol8-n2-2020-DelGiorgio-Girotto.pdf

Goldfrank B (2016). Los Procesos de "Presupuesto Participativo" en América Latina: éxito, fracaso y cambio. Revista de Ciencia Política / volumen 26 / № 2 / 2006 / 03 - 28. Universidad de Nuevo México, Estados Unidos.

Gutiérrez, G. (2014). Teoría de la toma de decisiones. Definición, etapas y tipos. Recuperado de https://www.gestiopolis.com/teoria-de-la-toma-de-decisiones-definicion-etapas-y-tipos/

Guzmán, M. (2014). Sistema de control de gestión y presupuesto por resultado: La experiencia chilena. Recuperado de https:/www.ucursos.cl/derecho/2011/1/D123A0632/3/material_docente/bajar?id_material=33 9970.

Koontz, H. (2014) Administración. Una perspectiva Global. México: Mc. Graw Hill.

Larrosa M. y Bermúdez E. (2020). Las Comisiones de Participación Ciudadana y la Consulta sobre Presupuesto Participativo en el contexto de la nueva Ley de Participación Ciudadana de la Ciudad de México en 2019. El Cotidiano; México City Tomo 36, N. ํ 224. Recuperado de https://www.proquest.com/openview/05e23e03b5982620134623815c03cc50/1?pqorigsite $=$ gscholar\&cbl $=28292$

Meza, C. (2018). La participación ciudadana como mecanismo de control social en el Perú: 


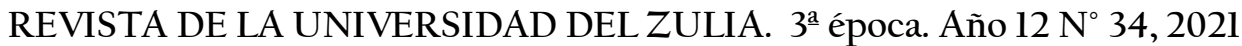

M. F. Urbina Cárdenas et al. /// Presupuesto participativo por resultados y la toma decisiones ... 185-205

DOI: http://dx.doi.org/10.46925//rdluz.34.12

Caso Contraloría General de la República. Recuperado de http://repositorio.ucv.edu.pe/handle/UCV/29363?locale-attribute=es

Pinochet, O, (2017). Participación ciudadana en la gestión pública local: el caso de la comuna de Pudahuel.

Recuperado

de http://repositorio.uchile.cl/bitstream/handle/2250/145052/Tesis\%20MGGP\%20$\% 20$ Osvaldo\%20Pinochet..pdf?sequence=1\&isAllowed $=y$

Quintero, S. (2013). Presupuesto Participativo en Bogotá. Recuperado de https://repository.javeriana.edu.co/bitstream/handle/10554/9012/QuinteroGalindoSandraIv onneth2013.pdf?sequence=1\&isAllowed=y

Reyes, J (2020). “ Participación ciudadana y gestión del presupuesto participativo en la Municipalidad Distrital de Querecotillo" (tesis posgrado). Universidad César Vallejo, Perú. Recuperado https://alicia.concytec.gob.pe/vufind/Record/UCVV_95bcfl69638bf3c4caa46928a02c8200

Rodríguez, J, (2017). Participación de los Actores Sociales en el Presupuesto Participativo año 2017, del distrito de Sabandía, Arequipa Perú. Recuperado de http://tesis.pucp.edu.pe/repositorio/bitstream/handle/20.500.12404/12380/RODR\%C3\%8D GUEZ_FERN\%C3\%8INDEZ_PARTICIPACION_DE_LOS_ACTORES_SOCIALES_EN_EL_ PRESUPUESTO_PARTICIPATIVO.pdf?sequence=1\& $\&$ isAllowed $=y$

Ruiz, I, (2016). El presupuesto participativo y su contribución en el cumplimiento de los objetivos institucionales de la provincia de Chepén - 2014". Recuperado de http://dspace.unitru.edu.pe/bitstream/handle/UNITRU/4909/ignacioruiz_sheyla.pdf?seque nce $=1 \&$ is Allowed $=y$

Quispe Mamani, J. C., Quispe Mamani, F., Guevara Mamani, M., Arce Coaquira, R. R., Yapuchura Saico, C. R., \& Catachura Vilca, A. (2021). Inversión pública y pobreza monetaria en las familias de la Region de Puno - Perú (2004 - 2019). Journal of the Academy, (4), 124-140. https://doi.org/10.47058/joa4.7

Secretaría de Gestión Pública. Presidencia del Consejo de Ministros del Perú (2021). Política Nacional de Modernización de la Gestión Pública al 202. Recuperado de http://www.pcm.gob.pe/wp-content/uploads/2013/05/PNMGP.pdf

Valverde K., Gutiérrez E., y García, F. (2013). Presupuesto público sin participación ciudadana. La necesidad de un cambio institucional en México para la consolidación democrática. Universidad Autónoma Nacional de México. Recuperado de http://www.revistas.unam.mx/index.php/rmcpys/article/view/42662

Vallejos, C. (2015). Innovación Democrática y Empoderamiento: Estudio Comparado de Ocho Experiencias de Presupuesto Participativo en Chile. Recuperado de https://www.tdx.cat/bitstream/handle/10803/285354/cavldel.pdf?sequence=1 